\title{
Double-Arm Clasp with Anterior Placed Occlusal Rest Retained Mandibular Distal Extension Removable Partial Denture - Stress Analysis and Alveolar Bone Height Changes of Abutments.
}

\author{
${ }^{*}$ Wageh $\mathrm{H}^{1}$, Abd El-Khalik $\mathrm{MM}^{2}$, Hegazy SA ${ }^{3}$, Mostafa $\mathrm{AZ}^{4}$ \\ ${ }^{1}$ BDS, MSD, Senior Teaching Assistant of Prosthodontics, Faculty of Dentistry, Mansoura University, Egypt. \\ ${ }^{2}$ BDS, MSD, Phd, Professor of Prosthodontics, Faculty of Dentistry, Mansoura University, Egypt. \\ ${ }^{3}$ BDS, MSD, Phd, Professor of Prosthodontics, Faculty of Dentistry, Mansoura University, Egypt. \\ ${ }^{4}$ BDS, MSD, Phd, Assistant Professor of Prosthodontics, Faculty of Dentistry, Mansoura University, Egypt. \\ Corresponding author: *Heba Wageh Abo-Zaed Elsaed.
}

\begin{abstract}
:
Aim: study the effect of double-arm clasps as retainer units for bilateral mandibular distal extension removable partial denture regard: Study of stress analysis applied on the abutments (in-vitro) and abutment alveolar bone height changes (in-vivo).The retainer clasps includes: Reverse circlet clasp and Modified Half-and-Half clasp assembly.

Materials and methods: In-vitro study: Model RPD distal extension with three frameworks of different retainer units for stress analysis. In-vivo study: In a randomized controlled clinical trial, fifteen patients with completely edentulous maxilla and remaining mandibular eight anterior teeth including 1st premolar. They were randomly divided to three groups. All patients received maxillary complete denture. Group I: where distal extension RPD designed with Reverse circlet clasp. Group II: where distal extension RPD designed with modified half- andhalf clasp assembly without distal occlusal rest and retentive clasp arm arising from anterior occlusal rest. Group III: where distal extension RPD designed with modified Half and Half clasp assembly without distal occlusal rest and modified retentive clasp arm arising from proximal guiding plate. The abutment tooth was evaluated radio graphically by digital periapical radiographs after insertion, after 6 months and after 12months after denture insertion.

Results: Group I (Reverse circlet clasp) significant increase in bone resorption of abutment compared to those in Group II and Group III (modified half-and-half clasp assembly). From stress point of view, in bilateral loading group I applied more stress to abutment compared to group II and III.

Conclusion: Group II \&III modified half-and-half clasp assemblies (stress releasing clasp assembly) superior to reverse circlet clasp regard to abutment alveolar bone resorption of distal extension cases. Reverse circlet clasp (Group I) not purely stress releasing by disengagement regarding double arm clasp utilize for this purpose. From stress transmitted point of view (bilateral loading), modified half-and-half clasp assembly (group II) exhibit less stresses transmitted to the abutment than exhibit in group III as a result obtained in this study (in-vitro) that minimize abutment alveolar bone resorption compared to group I to group II\& III. This research recommends the application of a new stress releasing clasp designs for distal extension RPD (modified halfand-half clasp assemblies as in group II \& III). These assemblies should be added to retainer units of stress releasing action by disengagement.
\end{abstract}

Keywords: mandibular, retainer, reverse, half-and-half, circlet, clasp assembly.

\section{Introduction}

Bilateral distal-extension removable partial denture is can be defined as a removable partial denture that is supported and retained by natural teeth only at one end of denture base segment and in which a portion of functional load is carried by residual ridge. ${ }^{1}$ The bilateral distal extension removable partial denture presents a treatment problem to the dentist. A major factor to be considered with regard to such partial dentures is of their composite support, coming from two entirely different oral structures which are teeth and residual ridge. If support can be utilized in such a manner that neither the teeth nor the residual ridge is abused the basis for a successful removable partial denture is established. However, if the supporting teeth or soft tissues are not used correctly and completely, consequent the result will be both mobility of abutment teeth and resorption of the residual ridge. ${ }^{2}$ Stress releasing clasps by disengagement can be classified in to: Stress releasing clasp of double arm clasp with anterior placed occlusal rest including:-Reverse circlet clasp (Reverse Aker's) and modified halfand- half clasp assembly. Stress releasing clasp of single arm clasp with anterior placed occlusal rest including:RPI clasp, RPA clasp ${ }^{3}$ and RPH clasp . ${ }^{4}$ 
Reverse circlet clasp is basically circumferential clasp but the occlusal rest placed mesially. It can be used in distal extension base because it control stresses transmitted to abutment. As denture base is depressed under function, the retentive clasp tip rotate gingivally to enter a greater amount of undercut and reduce the torsional stresses transmitted to the abutment tooth. ${ }^{5}$ A modified RPA clasp assembly design was developed to solve the problem of RPA clasp assembly, especially when the lower $1^{\text {st }}$ premolar was used as a distal - extension RPD abutment. Its design includes a mesial rest, a distal guiding plate, a buccal bracing /retentive arm and a lingual bracing arm. A mesial rest seat is prepared in the tooth occlusal table in its depressed mesiolingual portion. The mesial rest and the proximal plate are connected by a lingual bracing arm, cancelling the need for a separate minor connector. The lingual bracing arm is allowed to move tissue ward freely in this design during rotational movement of the distal extension saddle around the fulcrum line which passes through the mesial rests. ${ }^{6}$

Abd El-Khalik; et $\mathrm{al}^{7}$ concluded that; from occlusally approaching view of use the torqueing releasing clasp assemblies. Modified clasp may be the clasp of choice to decrease abutment alveolar bone resorption in the tooth - tissue supported prosthesis. Half-and-half stress releasing clasp assembly was suggested in this research as a new clasp system to retain abutment of distal extension RPD as a goal to utilize distobuccal retentive undercut (near to the distal extension case).This clasp assembly realizes the proper ideal requirements of circumferential clasp that retained abutment teeth of removable partial denture.

Stress releasing clasps by flexibility can be classified into:-Double arm clasp with posterior placed occlusal rest includes: combination clasp, RPL clasp and extended arm clasp. ${ }^{6}$ Single arm clasp with posterior placed occlusal rest includes: back action and reverse back action clasps.

\section{Materials And Methods}

In-vitro study: Model RPD distal extension with three frameworks of different retainer units for stress analysis. Applied load on RPD: Two loading protocol was carried out to estimate stresses applied to abutment teeth with different design retainer system these include:-Unilateral loading position and Bilateral loading position. Bilateral (central) loading: on the model, the bilateral load (100 N of $1 \mathrm{~mm}$.min speed) was applied on removable partial denture by using metal bar which was cut to be extended from the bucco-occlusal surface of the $1^{\text {st }}$ molars in one side to the other side of the model. The bar was then cemented into position by epoxy resin. The mid-point of the bar was marked as the loading point. At least 5 minutes were allowed as a period of rest between each reading to give a chance for heat dissipation from the grids of the strain gauge. These procedures were carried out for three designs. The readings were collected in micro-strains the arithmetic mean and slandered deviation of the recorded readings (micro-strains) after each loading points were calculated, tabulated, and statistically analyzed.

In-vivo study: Fifteen patients were selected for this study from the Prosthodontics Department, Faculty of Dentistry, Mansoura University according to the following criteria:-They were healthy with no systemic diseases relating to bone resorption which achieved through clinical examination by physician. They were with angle's class I maxillo-mandibular relationship and have maxillary completely edentulous arch against mandibular bilateral distal extension ridges posterior to first premolar. Edentulous ridges were firm and well formed. The space between the gingival margin of the remaining natural teeth and functional depth of floor of the mouth will not be less than $8 \mathrm{~mm}$.

For every patient the following were performed:-Diagnostic periapical radiograph for first premolar on each side was done. Maxillary and mandibular preliminary impression were made with irreversible hydrocolloid impression material and poured in dental stone.

After border molded auto polymerized acrylic resin custom tray maxillary secondary impression was made with ZOE. The lower diagnostic casts were surveyed and the distal extension RPD was designed as follow: Lingual bar which extended from right to left mandibular first premolar to connect bilateral distal extension bases. Clasp design: The patients were classified into three equal groups according to different designs of clasp assembly as follow: Group I: where distal extension RPD designed with Reverse circlet clasp. Group II: where distal extension RPD designed with modified half - and- Half clasp assembly without distal occlusal rest and retentive clasp arm arising from anterior occlusal rest. Group III: where distal extension RPD designed with modified Half and Half clasp assembly without distal occlusal rest and modified retentive clasp arm arising from proximal guiding plate.

After making mandibular altered cast impression, jaw relation was registered and try in denture was processed. Occlusal analysis was carried out. Digital periapical x-ray film of abutment teeth was performed at three observation periods.

Standardization of the peri-apical radiograph was performed as follows:-A standardized plastic film holder was used, so that; at one end it could be precisely arm attached and removed from cathode tube of $\mathrm{x}$-ray machine, at the other end, the extension arm contained a receiptable for the placement of a periapical film and a plastic baking the target film distance was fixed at $30 \mathrm{~cm}$. Autopolymerizing acrylic resin template was 
constructed to hold the film and plastic baking. The template was designed to be seated on the occlusal surfaces of the abutment and canine teeth on the patient's cast. This device was used to align parallel to the abutment tooth on the patient cast. The surface of the template facing the upper jaw arch was shaped to a tray for receiving rubber impression base impression material that relate to the upper arch while the patient close his jaw. ${ }^{\mathbf{8}}$ A stainless steel wire was embedded vertically in the acrylic template for measure the radiographic magnification errors (R.M.E.) . ${ }^{9}$ The patient was seated in upright position with head immobilized. The patient was guided to hold the template in the same way for the three X - ray exposures: T0: immediately at time of denture insertion. T6: 6 months after denture insertion. T12: 12 months of denture insertion.

Measuring of abutment interdental alveolar bone height:-Direct digitalization of measurement data was done using Corel Drow11 computer program. To eliminate the radiographic magnification error, scale of measurement was adjusted according to actual length of stainless steel wire. Abutment interdental alveolar bone height was determined by measuring the distance between cemento-enamel junction (CEJ) and interdental alveolar bone crest. . $^{10}$

\section{Radiographic reference lines and points for measuring abutment alveolar bone height change:}

Reference lines:-A line I connect the mesial (a) and the distal (b) represent the cemento-enamel junction of the abutment tooth (mandibular $1^{\text {st }}$ premolar). A line II representing the long axis of the abutment tooth (from root apex (c) passing through the midpoint (d) of the distance between mesial and distal CEJ; a-b distance). Reference points:- A\&B: were the highest points of the mesial and distal interdental alveolar bone of the mandibular $1^{\text {st }}$ premolar. C\&D: obtained by drawing two lines from A and B points paralleling to line II and crossing line $\mathrm{I}$ at $\mathrm{C}$ and $\mathrm{D}$ points respectively.

The mesial (A-C) and distal (B-D) radiographic distance were measured with Corel Draw 11.

The mean radiographic alveolar crest distance was equal:

$$
\frac{(\mathrm{A}-\mathrm{C}) \text { distance }+(\mathrm{B}-\mathrm{D}) \text { distance }}{2}
$$

The RME (Radiographic Magnification Error) was calculated according to Weiss and Rosien ${ }^{11}$ as follow:

$\mathbf{R M E}=\underline{\text { Actual length of the wire }(\mathrm{M})}$

Radiographic length of the wire (m).

The actual C E J - alveolar crest distance was calculated by multiplying the R M E by radiographic C E J alveolar crest distance

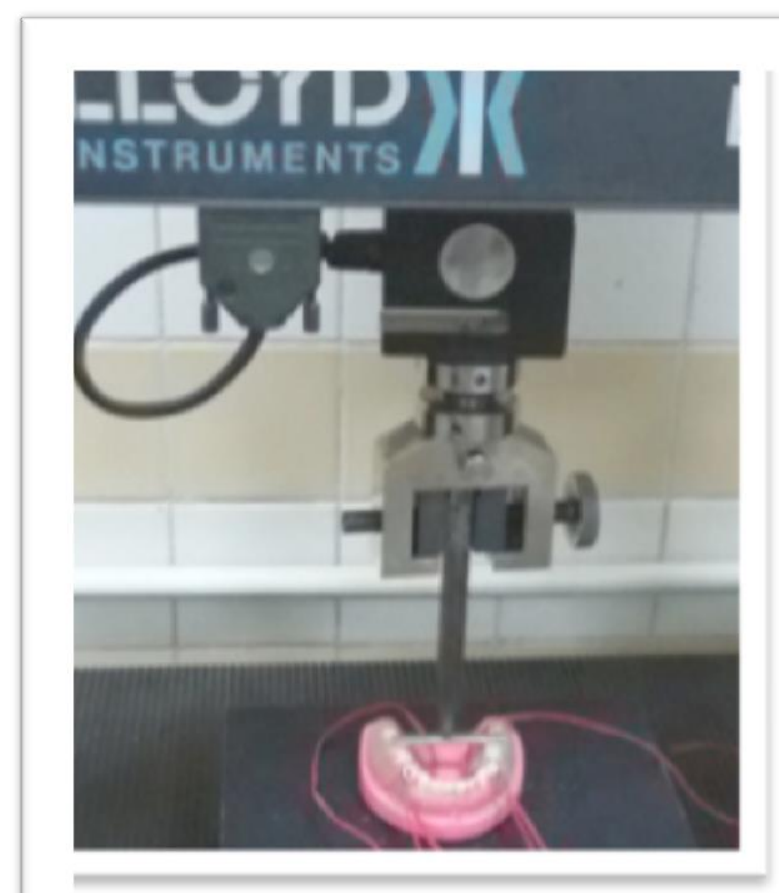

Fig 1: Bilateral (central) static loading of the model with strain gauge

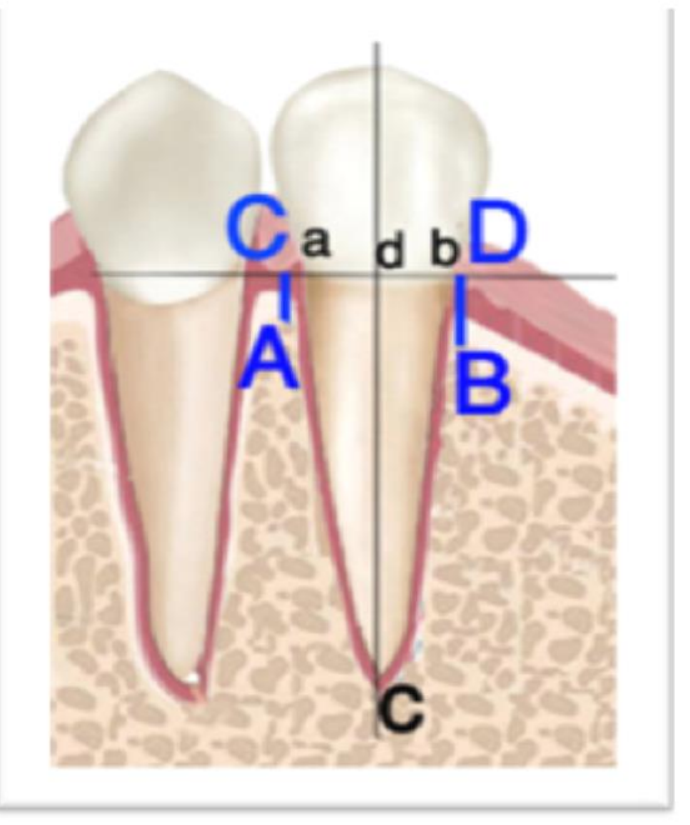

Fig 2: Diagram shows reference lines \& points for measuring abutments alveolar bone height 


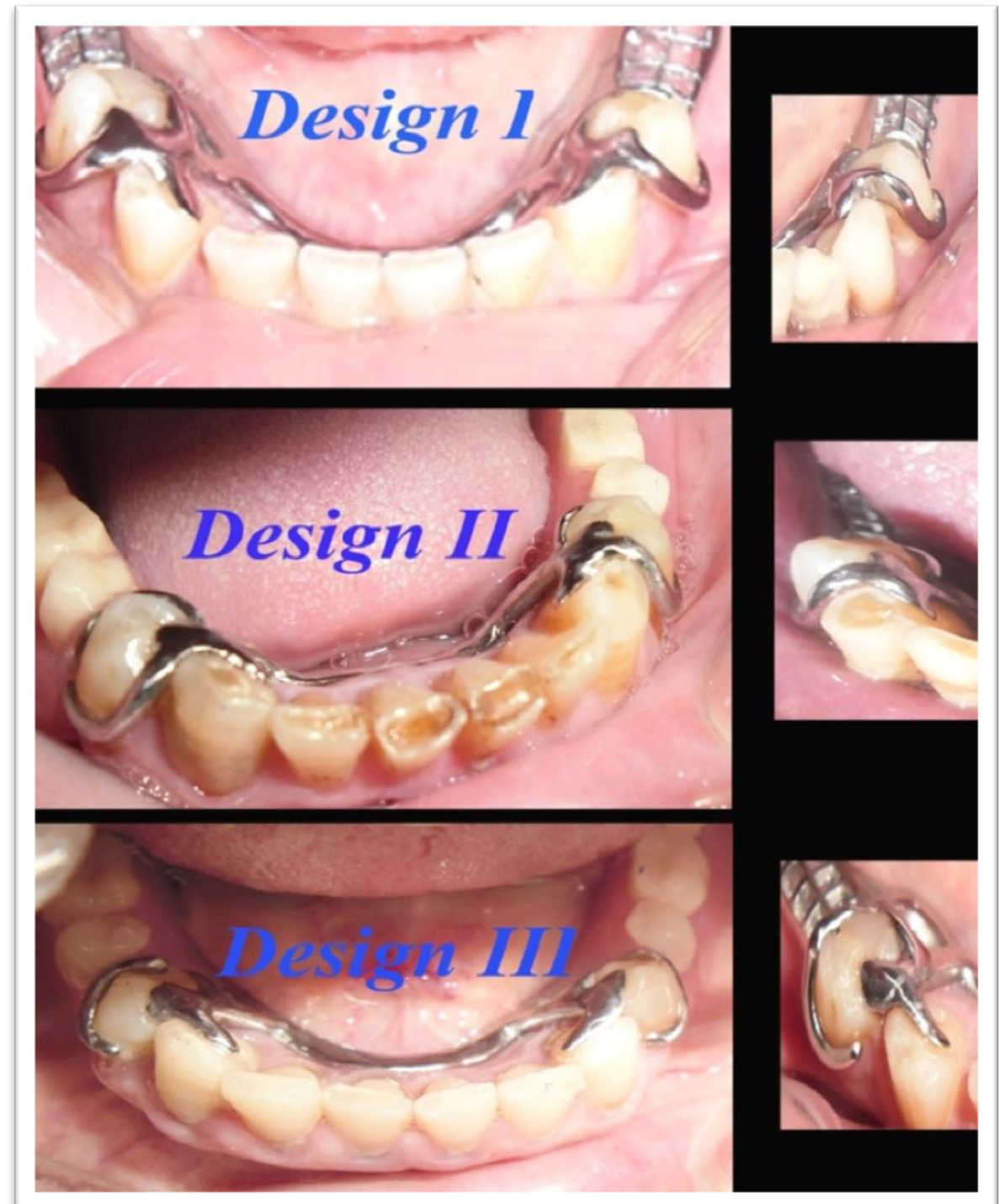

Fig 3: Different retainer units used to retain mandibular distal extension RPDs.

\section{Results}

The in-vitro study was evaluated and analyzed statistically to evaluate the mean micro-strains values for three designs (I, II, III) after application of bilateral (central) static loading (100 N ). There was significant difference between three designs in the mean amount of stresses applied on to the abutment where $\mathrm{F}=4.10$ and $\mathrm{LSD}=9.865$ and $\mathrm{P}=0.0217$. Table (1)

The in-vivo study was evaluated and analyzed by Anova test for determining the significant difference of abutment alveolar bone resorption of three groups I, II, III along the follow-up periods. There was significant difference between the studied groups in the mean amount of abutment crestal alveolar bone resorption where $\mathrm{P}=0.01$ and $0.07^{*}$ and $0.001 *$ for the first, the second interval period and total 12 months of RPD insertion respectively. Table (2)

Table1: Show the mean micro-strains values for three designs (I, II, III) after application of bilateral (central) static loading $(100 \mathrm{~N})$.

\begin{tabular}{|l|l|l|l|l|l|}
\hline & Mean & S.D & LSD & F value & P value \\
\cline { 1 - 3 } Design I & $51.50 \mathrm{a}$ & 16.23 & 9.865 & 4.10 & $0.0217^{*}$ \\
\cline { 1 - 3 } Design II & $38.50 \mathrm{~b}$ & 16.94 & & & \\
\cline { 1 - 3 } Design III & $40.25 \mathrm{~b}$ & 13.33 & & & \\
\hline
\end{tabular}


Table 2: Anova test for determining the significant difference of abutment alveolar bone resorption between groups I, II, III along the follow up period.

\begin{tabular}{|c|c|c|c|c|c|}
\hline Variable & \multicolumn{2}{|c|}{ Abutment crestal alveolar bone resorption of studied groups } & \multirow{2}{*}{ F Value } & \multirow{2}{*}{ P Value } \\
\hline & Group I & Group II & Group III & & \\
\hline $\mathbf{1}^{\text {st }} \mathbf{6}$ months & $0.123 \pm 0.011$ & $0.132 \pm 0.021$ & $0.159 \pm 0.015$ & 6.231 & $0.01^{*}$ \\
\hline $2^{\text {nd }} \mathbf{6}$ months & $0.15 \pm 0.016$ & $0.129 \pm 0.017$ & $0.158 \pm 0.021$ & 3.283 & $0.07^{*}$ \\
\hline Total 12 months & $0.273 \pm 0.019$ & $0.261 \pm 0.020$ & $0.317 \pm 0.009$ & 14.989 & $0.001^{*}$ \\
\hline
\end{tabular}

*Significant $\mathrm{P} \leq 0.05$

\section{Discussion}

In this research, abutment interdental alveolar bone resorption was significantly observed along the interval periods of the study after insertion of mandibular distal extension removable partial denture. The result was revealed that, there was significant difference in each three groups (I, II, III) in all interval periods except $1^{\text {st }}$ six months in group I there is insignificant difference. The results of abutment interdental alveolar bone resorption was agreed with the results of stresses measured by a strain gauge, as there was found that, the presence of stresses between three groups (I, II, III) .

As the denture base move under function loading, the abutment teeth subjected to stresses that released by disengagement that provided by a stress releasing clasp principles. Because the load transmitted to the abutment tooth is not within its physiologic limits, abutment alveolar bone resorption will be exhibited along the interval periods of the study. Carr et $\mathbf{a l}^{\mathbf{1 2}}$ stated that: the terminal third of clasp arm that engages the abutment undercut away from edentulous area, if it is used in tooth-tissue supported RPD, rotation occurs during tissue ward movement and the retentive clasp terminal will engage more undercut creating excessive stresses to the abutment.

In this study, group I exhibit significantly greater abutment interdental alveolar bone resorption when it compared either with group II and III. When comparing group II and III there was insignificant difference of abutment interdental alveolar bone resorption along the interval periods and follow up periods of the study.

This may be explained that; in modified half-and-half clasp assemblies group II \& group III the retentive arm move independently from the reciprocal arm (both arm of group I and group II not joined together to interproximal minor connector) under functional loading of the denture base. Phoenixet $\mathbf{a l}^{\mathbf{1 3}}$ stated that: the denture base is displaced under functional loading, the retentive clasp tip rotate gingivally to engage great amount of retentive undercut and decrease the torsional stress to the abutment teeth. Due to horizontal reciprocation of the modified clasp assembly design provides better encirclement, two arm clasps, that prevent the tooth from moving out of confines of the clasp assembly. Hakkoum; ${ }^{\mathbf{1 4}}$ mentioned that; the existence of a bracing arm above the height of contour may preclude the downward movement of the retentive tip. So, it would not disengage the tooth when an excessive load is applied to the distal extension base. Moreover, the role of the mesial rest may be cancelled so the harmful forces are transmitted to the abutment.

Consequently, more stresses applied to the abutment teeth by reverse circlet clasp compared by modified half-and-half clasp assembly either retentive arm placed mesially or distally away from the saddle causing excessive abutment alveolar bone resorption with the former compared to the other one. This explanation was reinforced by the in-vitro results obtained in this study. Where the bilateral (central) loading and unilateral loading (right) side in group I exhibit more stresses than in group II and group III.

Firstly, this may explained that, this result found that there was insignificant difference in abutment alveolar bone resorption when compared with modified half-and-half clasp assemblies that utilized the retentive undercut mesially to that utilized the retentive undercut distally. This result indicated that, the location of retentive undercut either mesially or distally is not a factor in selecting modified half-and-half clasp assembly as a retainer unit. As EI-Shoukouki ; ${ }^{15}$ studied analysis and comparison of stress transmission characteristics on abutment teeth induced by different clasp designs for distal extension RPD, he found that the occlusally approach torqueing releasing clasp assembly utilize the RPA design induced more stresses along the distal abutments when these stresses were compared to that provided by RPI clasp assembly design.

In this study, modified half-and-half clasp assembly utilized mesially placed occlusal rest and retentive arm that engage a retentive undercut near to the saddle may be suggested as a new stress releasing clasp design for distal extension RPD. It should be added to retainer units of stress releasing action by disengagement.

Secondly, the greater stresses in group III this may be due to bracing arm originating from the primary occlusal rest in group III will be the cause of greater stresses to the abutment teeth so, when the prosthesis is rotated greater force will be subjected to the area of the clasp that located to the area above survey line even designing the bracing arm to allow free tissue ward movement and superior border of the bracing arm contact the area with survey line. This explanation will be reinforced by the result obtained by greater stresses of group 
III more than group II in addition to abutment alveolar bone resorption in group III more than group II even the result of study, there is no significant result.

Consequently, according to this explanation, the author pointed out that, the stresses transmitted from the bracing arm originating from the primary rest isn't comparable to that stresses transmitted by the bracing arm where the bracing arm originating from the proximal plate because the bracing arm of group II freely totally moved gingivally compared to that of groups I and III. A further investigation will be carried out to study the effect of the bracing arm to be in contact or not to the primary occlusal rest regard to stress transmitted to the abutment.

This study revealed that an appropriate close approximation of the mean amount of abutment alveolar bone resorption that was observed during the $1^{\text {st }}$ and $2^{\text {nd }}$ interval periods of study. This may be due to the bracing component of double arm clasp assembly of stress releasing action positioned at the junction between middle and gingival third of the abutment teeth that minimize stress transmitted to the abutment along the interval periods of the study. Boucher and Renner; ${ }^{16}$ stated that; the bracing component which performs the function of stabilization, reciprocation and encirclement should be placed at the junction between middle and gingival third of the abutment teeth. Encirclement is provided by entire clasp assembly that contact tooth surface for more than 180 degree, it prevents tooth from moving away from the clasp assembly or prevents the clasp assembly from slipping off tooth surface when it is subjected to vertical, horizontal and torqueing forces.

close approximation of stresses (bilateral loading) transmitted to the abutment teeth either using stress releasing clasp assembly that utilized mesial or distal retentive undercut even a significant result was found when comparing of group II and group III together (group II less stresses compared to group III). Bilateral loading representing dynamic function and there is close approximation with in-vivo study (Bilateral loading coincide with clinical study).

\section{References}

[1]. Academy of Prosthodontics: Glossary of Prosthodontic terms. J prosthet Dent 2005; 94(1):10-92.

[2]. Anand U, Pradeep N, Naveen S, et al: A Comparative Study of Impression Procedures for Distal Extension Removable Partial Dentures.J of Contemp Dent Pract, 2011; 12(5):333-338.

[3]. Phoenix RD, Cagna DR, DeFreest CF. Stewart's Clinical Removable Partial Prosthodontics, 4th edition. Quintessence, Illinois. 2008:18-510.

[4]. NazarovaE, Taylor TD: The RPH Clasp Assembly: A Simple Alternative to Traditional Designs, Journal of Prosthodontics 21 (2012) 331-333.

[5]. Stewart KL, Rudd KD and Kuerbker WA: Clinical removable partial Prosthodontics. $1^{\text {st }}$ ed. St. Louis, Toronto, London. 1983.

[6]. Shifman A, Ben-Ur Z: The mandibular first premolar as an abutment for distal - extension removable partial denture : a modified clasp assembly design. Br Dent J 2000; 188(5): 246-8.

[7]. Abd El-Khalek MM, SaadAldeenMA ,ElMekkawy NS: Stress Releasing Clasp Assemblies Design Related To Abutment Alveolar Bone Resorption. Egyptian Dental Journal.2006; 52:1785.

[8]. El-Charkawi HG: Residual ridge changes under titanium-plasma-sprayed screw implant systems. J Prosthet Dent 1989; 62(5): 576580 .

[9]. El-Basty SA: The effect of the position of occlusal rest on the mobility and periodontal condition of the abutment teeth, in bilateral free end saddle cases Ph. D. Thesis. Cairo University, 1982.

[10]. Hausmann E, Allen K, Christersson L, Genco RJ: Effect of X-ray beam vertical angulation on radiographic alveolar crest level measurement. J Periodontal Res. 1989;24(1):8-19.

[11]. Weiss MB, Ronen E: New device to quantitate alveolar bone loss. Oral Surg, Oral Med, Oral Path. 1977;44(2):322-4.

[12]. Carr AB, McGivney GP, Brown DT: McCracken's Removable Partial Dentures, $11^{\text {th }}$ ed 2004; 80,106.

[13]. Phoenix R. D., Cagna D. R., DeFreest C. F: Stewart's clinical partial prosthidontics. 3rd ed., Chicago, Quintessence publishing Co. Inc., P63-71, 74-75, 284 (2003).

[14]. Hakkoum MA:New Clasp Assembly for Distal Extension Removable Partial Dentures: The Reverse RPA Clasp Journal of Prosthodontics 25 (2016) 411-413

[15]. El-Shoukouki AH:Analysis and comparision of stress transmission characteristics on abutment teeth induced by different clasp designs for distal extension removable partial denture. Egyp Dent J 1996; 42: 2075-2084.

[16]. Boucher LJ, Renner RP: Treatment of partially edentulous patients. (6 ${ }^{\text {th }}$ ed) St Louis, Toronto, London C.V. Mosby Co. 1982, p: $24,26,64,67,76,77,139$.

*Wageh H. "Double-Arm Clasp with Anterior Placed Occlusal Rest Retained Mandibular Distal Extension Removable Partial Denture - Stress Analysis and Alveolar Bone Height Changes of Abutments." IOSR Journal of Dental and Medical Sciences (IOSR-JDMS) 16.7 (2017): 114-119. 